\title{
Die Bestimmung des Atomgewichtes der Elemente der seltenen Erden.
}

\author{
Von \\ W. Feit und K. Przibylia.
}

Bis vor kurzer Zeit galt als die einzige sichere Methode zur Atomgewichtsbestimmung der Elemente der seltenen Erden die alte, von Bunsen angegebene sogenannte Sulfatmethode, welche darin besteht, dafs man eine gewogene Menge des Oxyds in das wasserfreie neutrale Sulfat überführt, und aus der Gewichtszunahme das Atomgewicht berechnet. Im Laufe unserer Arbeiten haben wir Gelegenheit gehabt, uns von der Richtigkeit der Behauptung von Brauner und Pavlicek, ${ }^{1}$ dals diese Methode mit einem nur sehr schwer zu überwindenden Fehler behaftet ist, zu überzeugen. Wir setzen als bekannt voraus, dafs, wie von BRAUNER und neuerdings von $\mathrm{WILD}^{2}$ und BRILL $^{3}$ gezeigt wurde, die von KRÜss zum Verjagen der freien Schwefelsäure angegebene Temperatur von $350^{\circ}$ viel zu niedrig ist. Es ist eigentümlich, dafs, während die basischen Sulfate von der Formel $\mathrm{R}_{2} \mathrm{O}_{3} \mathrm{So}_{3}$, welche vor kurzem von BRILu untersucht wurden, eine Zersetzungstemperatur zeigen, welche, wie ja zu erwarten war, mit abnehmender Basizität niedriger wird, diese Temperaturen bei den normalen Sulfaten in gar keinem Zusammenhange mit der Stärke der Basizität zu stehen scheinen. Diese Beobachtungen Britus werden durch einige von uns angestellte Versuche bestätigt, z. B. verträgt von den normalen Sulfaten des Lanthans, Yttriums und Ytterbiums das der schwächsten Base, des Ytterbiums, die höchste Temperatur. Aus diesem Grunde glauben wir, dals die Vermutung

1 Transactions of the Chemical Society 81 (1902), 1243.

2 Z. anorg. Chem. 38, 191.

3 Z. anorg. Chem. 47, 464.

z. anorg. Chem. Bd. 50 . 
Brauners, der Fehler bei der Bestimmung des Atomgewichtes nach der Sulfatmethode wachse mit abnehmender Stärke der Basizität, nicht gerechtfertigt ist. Offenbar hängt die Darstellung absolut neutraler Sulfate auf dem hier in Betracht kommenden Wege, dem Verjagen der überschüssigen Schwefelsäure bei höherer Temperatur, mit der Zersetzungstemperatur der sauren Sulfate, deren Kenntnis wir den Untersuchungen von BRAUner und PIOEK ${ }^{\mathfrak{l}}$ verdanken, zusammen.

Bei der Bestimmung des Atomgewichtes des Lanthans korrigierte BRAUNER ${ }^{2}$ den durch die saure oder alkalische Reaktion des Sulfats bedingten Fehler dadurch, dals er das Sulfat nach dem Wägen in Wasser löste, die Lösung mit Methylorange versetzte und das Plus oder Minus an Säure durch Titration mit $n / 20$ Alkali oder $n / 20$ Säure ermittelte. BRAUNER bemerkt jedoch, dafs sich ein etwa noch vorhandener minimaler Wassergehalt des Sulfats nicht feststellen läfst. Dieser Gehalt an Wasser kann nun zweierlei Ursachen haben, falls das Sulfat noch sauer reagierte. Einmal kann die vorhandene Schwefelsäure als Anhydrid, aber auch als Hydrat zugegen sein; andererseits aber ist durch nichts bewiesen, dafs das Sulfat nicht noch einen geringen Gehalt an Kristallwasser besitzt, welcher erst bei einer Temperatur fortgehen würde, bei welcher das normale Sulfat schon zum Teile zersetzt ist. Reagierte die Lösung des Sulfats neutral oder alkalisch, so ist nur der letzte Fall zu berücksichtigen. Ähnliche Verhältnisse fand RichaRds ${ }^{3}$ bei der Analyse des Kupfersulfats behufs Bestimmung des Atomgewichtes dieses Elementes.

Vielfach werden, besonders in neuerer Zeit, die Atomgewichtsbestimmungen durch Überführen von neutralem Sulfat in Oxyd ausgefübrt oder, wie z. B. von URBAIN durch Überführen des achtfach gewässerten Sulfats in das wasserfreie Sulfat und weiteres Verjagen der Schwefelsäure bis zum Oxyd. Da es leicht ist, ein absolut neutrales Sulfat herzustellen, so fällt bei dieser Methode der eine Fehler völlig fort; bei der UnbaIsschen Modifikation hat man noch dazu den Vorteil, mit derselben Menge eingewogener Substanz zwei voneinander unabhängige Atomgewichtsbestimmungen, welche auf verschiedener Basis beruhen, ausführen zu können, welche sich da. her gegenseitig kontrollieren. Immerhin aber hat man keinen An-

1 Z. anorg. Chem. 38, 322.

2 Transactions of the Chemical Society 81 (1902), 1252.

${ }^{3} Z$. anorg. Chem. 1, 179. 
halt dafür, dafs auch wirklich das angewandte Sulfat genau $8 \mathrm{Mol}$. Wasser enthält; andererseits weifs man nicht, ob das zur Wägung gebrachte wasserfreie Sulfat wirklich neutral (d. h. nicht alkalisch) und absolut wasserfrei ist. Es könnte nun eingewendet werden, dals man ja durch die Wägung des Oxyds eine Kontrolle der ersten Bestimmung hat. Es ist aber demgegenüber zu bemerken, dafs sich bei der Kleinheit der Fehler, um die es sich hier handelt, eine genügende Übereinstimmung zeigen kann, selbst wenn die soeben als möglich angedeuteten Fehler wirklich gemacht werden, zumal da es kein Mittel gibt, sie zu entdecken.

Es geht aus diesen Ausführungen hervor, dals die praktische Ausführung der Sulfatmethode mit nicht geringen Schwierigkeiten verknüpft ist, wenn die Resultate Anspruch auf Genauigkeit machen sollen. Rechnet man hierzu den erforderlichen grofsen Aufwand an Zeit, so erhellt, dals die Ausarbeitung einer einfachen und doch sicheren Methode von nicht zu unterschätzender Wichtigkeit ist. Wir denken dabei sowohl an exakte Atomgewichtsbestimmungen, als auch an die grofse Anzahl von Bestimmungen, welche sich bei Arbeiten über die Trennung der seltenen Erden als unumgänglich notwendig erweisen. Vor kurzem hat BRILL ${ }^{1}$ die Verwendbarkeit der NeRnst schen Mikrowage für derartige Bestimmungen empfohlen. Es handelt sich jedoch auch hier um die Überführung von Oxyd in Sulfat bezw. von Sulfat in Oxyd; die oben angeführten Schwierigkeiten sind also auch hier zu überwinden. Einen grolsen Vorteil würde die Verwendung von nur wenigen Milligrammen Substanz allerdings bieten, doch steht die erzielte Genauigkeit nicht im richtigen Verhältnisse zur angewandten Mühe; bei geringen Differenzen in den Atomgewichten der in Betracht kommenden Elemente ist daher die Verwendung dieser Methode nicht zu empfehlen, während sie z. B. zur Feststellung eines Gehaltes an Yttrium in einem Gemenge mit seinen Begleitern in manchen Fällen völlig genügen dürfte. Eine Reihe von Versuchen, welche wir angestellt haben, haben die Beobachtungen von Brauner, WiLd und Brilu bestätigt, dals es unter allen Umständen erforderlich ist, bei der Überführung von Oxyd in Sulfat die oben erwähnte Korrektur durch Titration der Lösung des gewogenen Sulfats mit Alkali bezw. Säure anzubringen. Es dürfte hiernach keinem $Z$ weifel unterliegen, dafs die von uns ${ }^{2}$ vor einiger

1. c.

2 Z. anorg. Chem. 43, 212. 
Zeit beschriebene Methode der Bestimmung des Atomgewichtes durch Auflösen einer gewogenen Menge des Oxyds in n/2 Schwefelsäure und Zurückmessen der überschüssigen Säure durch $n / 10$ Alkali unter Verwendung von Methylorange bezw. Äthylorange als Indikator zum richtigen Resultate führen mufs, da ja das Endresultat bei der Sulfatmethode nach BRAONER auch auf alkalimetrischem Wege gefunden wird. Hierzu kommt nun aber, dafs die oben gegen die Sulfatmethode angeführten Bedenken bei dieser Methode gar nicht in Frage kommen, vor allem der gar nicht kontrollierbare Wassergehalt des Sulfats. Später hat auch HermanN ${ }^{1}$ diese Methode beschrieben und die Übereinstimmung seiner Zahlen mit der Sulfatmethode nachgewiesen. Henmann nimmt statt Schwefelsäure jedoch Salzsäure; hierbei ist natürlich das Arbeiten mit Rückflufskühler erforderlich. Hermann zieht die Salzsäure der Schwefelsäure vor, weil die Lösung leichter erfolgen soll; nach unseren Erfahrungen trifft dies nicht zu, nur mufs man bei denjenigen Oxyden, welche sich erst nach längerem Erhitzen lösen und welche dabei leicht Sulfat abscheiden können, für eine angemessene Menge Wasser sorgen. Übrigens haben wir dies nur in Ausnahmefällen beobachtet.

Die Hauptrorbedingung der Ausführbarkeit der Methode ist natürlich die, dals das normale Sulfat des fraglichen Elementes in einer Lösung von einer Konzentration, wie sie bei der Bestimmung des Atomgewichtes zur Anwendung kommt, gegen Methylorange absolut neutral reagiert. Nachdem wir verschiedentlich festgestellt hatten, dals die Sulfate des Lanthans, Neodyms und Yttriums in einer Konzentration von etwa $1: 40$ dieser Bedingung entsprechen, unterwarfen wir die schwächste der bei uns in Betracht kommenden Basen, das Ytterbiumoxyd, derselben Prüfung. Angewandt wurde ein etwas Erbium enthaltendes Präparat mit dem Atomgewicht von nahezu 173.0. Das Oxyd wurde in möglichst wenig verdünnter Schwefelsäure gelöst, die Lösung zur 'Trockne verdampft und der Rückstand zum Verjagen der überschüssigen Schwefelsäure schwach geglüht. Die Lösung des so erhaltenen Salzes reagierte ganz schwach sauer; sie wurde mit reinem Alkohol gefüllt, der Niederschlag mit Alkohol gewaschen und bei $130^{\circ}$ getrocknet. $1 \mathrm{~g}$ des so erhaltenen Sulfates wurde in $40 \mathrm{ccm}$ Wasser gelöst und mit einigen Tropfen Methylorange versetzt. Die entstandene Färbung war rein gelb, genau wie sie durch reines Wasser hervorgebracht wurde und ein Tropfen einer $n / 10$ Schwefelsäure bewirkte das Erscheinen einer deutlich rötlichen Nuance.

1 Dissertation, München 1906, S. 21. 
Die für die Atomgewichtsbestimmungen von uns neuerdings benutzte kurzarmige BuNGEsche Wage zeigte selbst bei hoher Belastung deutlich 0.1 Milligramm an; die Bruchteile liersen sich noch schätzen, doch begnügten wir uns stets mit der Feststellung der vierten Dezimale. Der Nullpunkt der Wage blieb auch bei längeren Wägungsreihen konstant, eine diesbezügliche Kontrolle wurde häufig ausgeführt. Die Gewichte bestanden aus vergoldetem Messing, die Bruchgramme aus Aluminium. Der ganze Gewichtssatz war in 40, 30, 20, $10 \mathrm{~g}$ bezw. Dezigramm und Zentigramm gestückelt, sodals ein Stück von bestimmtem Nominalwerte nur einmal im Satze vorkommt. Bei den oft ausgeführten Kontrollen des Gewichtssatzes ergaben die einzelnen Stücke nur Abweichungen von Bruchteilen eines $1 / 10 \mathrm{mg}$ von ihrem Sollgewichte.

Als Titersubstanz zum Einstellen der Säure diente Natriumkarbonat, dargestellt aus Natriumbikarbonat pro analysi von MERCK. Um die Brauchbarkeit dieses Präparates zu prüfen, unterwarfen wir es einer Analyse. $\mathrm{Zu}$ dem Zwecke wurden $1.6740 \mathrm{~g}$ des beim Glühen im Platintiegel resultierenden Karbonats in zweimal destilliertem Wasser gelöst und der nach 24stündigem Stehen abgeschiedene Niederschlag auf einem kleinen, aschenfreien Filterchen gesammelt, gewaschen, verascht und stark geglüht. Sein Gewicht betrug $0.0006 \mathrm{~g}$. In einem Tropfen Salzsäure gelöst und mit Ammoniak gefällt, ergab sich ein Gehalt von $0.0001 \mathrm{~g}$, welcher als Eisenoxyd und Tonerde in Rechnung gezogen wurde, so dals $0.0005 \mathrm{~g}$ für Calciumoxyd übrig blieben. Das Filtrat der ersten Operation wurde mit Salzsäure übersättigt und in einer Platinschale zur Trockene verdampft. Beim Auflösen in Wasser blieb ein Rückstand von $0.0004 \mathrm{~g}$, welcher als Kieselsäure verrechnet wurde. Das Natriumkarbonat, welches übrigens völlig frei von Chlor und Schwefelsäure war, enthielt demnach folgende Verunreinigungen:

$$
\begin{array}{ll}
0.006 \% & {[\mathrm{FeAl}]_{2} \mathrm{O}_{3}} \\
0.053 \% & \mathrm{CaCO}_{3} \\
0.024 \% & \mathrm{SiO}_{2} .
\end{array}
$$

Wenngleich bei richtiger Verrechnung dieser Verunreinigungen sich dieselben als so gering herausstellen, dafs die dadurch hervorgerufenen Fehler innerhalb der Wägefehler fallen, so haben wir das Präparat doch gereinigt. Nach schwachem Glühen wurde das hinterbliebene Karbonat in einer Platinschale in Wasser gelöst, nach 24 Stunden die Lösung von dem geringen Niederschlage abfiltriert 
und die Lösung in der Platinschale zur Kristallisation verdampft. Nur die erste Kristallisation wurde benutzt. Die Kristalle wurden abgesaugt, die Mutterlauge mit etwas Wasser fortgewaschen und die Kristalle über Magnesiumchloridhexahydrat, welches uns in grofser Reinheit als Mineral zur Verfügung steht, getrocknet; schliefslich wurde es unter allmählicher Temperatursteigerung völlig entwässert.

Als Säure benutzten wir $n / 2$ Schwefelsäure, während zum Zurücktitrieren $n / 10$ Natronlauge verwendet wurde, welche nach dem Küsterschen Verfahren aus metallischem Natrium hergestellt worden war. Die Schwefelsäure wurde stets gewogen, nicht gemessen; hierdurch wurden die Ablesefehler, sowie die Änderung des Titers infolge der Temperaturveränderung so gut wie möglich eliminiert. Bei der fünffach schwächeren Natronlauge, von welcher übrigens stets nur wenige Kubikzentimeter verbraucht wurden, erachteten wir die Messung für völlig genügend, umsomehr als die nachträgliche Wägung bei der während der Ausführung der Titration nicht zu vermeidenden Verdunstung zu neuen Fehlern Veranlassung gegeben haben würde.

Zur Einstellung der Säure wurde zunächst das Natriumkarbonat, dessen Menge so bemessen wurde, dals sie nicht ganz $20 \mathrm{~g}$ Säure zu sättigen vermochte, in einem Platintiegel, welcher sich in einem Porzellantiegel befand, vorsichtig bis zur Gewichtskonstanz geglüht. ${ }^{1}$

Es sei hier bemerkt, dafs in der Regel nur zwei, selten drei Wägungen erforderlich waren, wobei die Differenz höchstens $0.1 \mathrm{mg}$ betrug. Die Wägungen geschahen nach völligem Erkalten im Schwefelsäure-Exsikkator, wozu wenigstens eine Stunde erforderlich war, im bedeckten Platintiegel, der sich jedoch nicht in einem Wägegläschen befand. Mit der Anwendung der Wägegläschen haben wir nämlich, da uns ein Raum mit völlig konstanter Temperatur nicht zur Verfügung stand, keine guten Erfahrungen gemacht. Die Schwefelsäure wurde in einem bedeckten ERLENMEYkRschen Kolben aus Jenaer Glas (gewöhnliche Gläser sind absolut unbrauchbar) abgewogen, wobei eine Wägung auf $1 \mathrm{mg}$ genau völlig genügt, alsdann nach vorheriger Verdünnung der Säure, von welcher stets $20 \mathrm{ccm}$ abgewogen waren, mittels reinen Wassers auf etwa $40 \mathrm{ccm}$ das Natriumkarbonat hinzugefügt und der Kolben vorsichtig erhitzt, bis die Kohlensäure so gut wie völlig ausgetrieben war. Nach dem Erkalten wurde Methylorange hinzugefügt und mit Natronlauge zurücktitriert, wobei als Vergleichslösung reines Wasser diente, welches

${ }^{1}$ Das Heizgas war aus Gasolin bereitet, es war daher völlig frei von Schwefel. 
durch unser Methylorange rein gelb gefärbt wurde. Als Beispiel einer Einstellung der Säure mögen die Zahlen in der folgenden Tabelle dienen; $(a=g$ Natriumkarbonat; $b=$ zur Neutralisation erforderliche Säure in $g ; c=g$ Natriumkarbonat, welche zur Neutralisation von $1 \mathrm{~g}$ nötig sind; $d=g$ Sauerstoff, welche durch $1 \mathrm{~g}$ Säure angezeigt werden.

\begin{tabular}{c|c|c|c|c}
\hline \hline $\mathrm{Nr}$. & $a$ & $b$ & $e$ & $d$ \\
\hline \hline 1 & 0.5350 & 20.381 & 0.026250 & 0.0039615 \\
2 & 0.5262 & 20.060 & 0.026231 & 0.0039586 \\
3 & 0.5238 & 19.994 & 0.026198 & 0.0039536 \\
4 & 0.5251 & 20.011 & 0.026241 & 0.0039601 \\
5 & 0.5237 & 19.984 & 0.026206 & 0.0039548
\end{tabular}

Im Mittel ist also $1 \mathrm{~g}$ Säure $=0.003958 \mathrm{~g}$ Sauerstoff. Hierbei ist angenommen, dals das Molekulargewicht des Natriumkarbonats gleich 106.02 ist. In der beschriebenen Weise wurde der Titer der Säure häufig kontrolliert, ebenso wie die Relation der gewogenen Menge Säure zur gemessenen Natronlauge.

Die Ausführung der Atomgewichtsbestimmungen gestaltet sich nun folgendermalsen. In einem Platintiegel wurde ein Quantum des betreffenden Oxyds, welches so grofs ist, dafs es etwa $18-19 \mathrm{ccm}$ der Schwefelsäure zu sättigen vermag, annähernd genau abgewogen und alsdann über einem grolsen mit Gasolingas gespeisten Teklubrenner eine halbe Stunde mit aufgelegtem Deckel geglüht. Der Tiegel befindet sich dabei in einer oben und unten offenen Kapsel, welche dadurch gebildet wird, dafs zwei abgestumpfte Kegelmäntel aus dünnem Eisenblech, welche innen mit Asbestpappe verkleidet sind, mit ihrer Basis aufeinandergesetzt werden. Die Asbestbekleidung wird noch mehrere Male mit einem Brei aus gebrannter Magnesia und Wasserglas verstrichen und jedesmal scharf getrocknet. In die untere Öffnung dieser Kapsel reicht das obere Ende des Brenners, in der Mitte ruht der Tiegel auf einem Dreieck, welches in der Kapsel leicht anzubringen ist und durch die obere Offnung entweichen die Verbrennungsgase. Infolge dieser Anordnung sind wir imstande, mit einem Einbrenner im geschlossenen Porzellantiegel in wenigen Minuten $20 \mathrm{~g}$ Kupfer zum Schmelzen zu bringen.

Der Tiegel mit Oxyd wird nach halbstündigem Erkalten im Schwefelsäure-Exsikkator bei aufgelegtem Deckel gewogen und alsdann abermals eine halbe Stunde geglüht. In der Regel zeigte 
die zweite Wägung Konstanz, doch nicht immer. Einzelne Oxyde scheinen nämlich die letzten Spuren von Kohlensäure nur sehr schwierig zu verlieren; in diesem Falle ist nochmaliges Glühen erforderlich. Das Wägen des Tiegels geschah auf der Wage bei geschlossenem Schieber, ohne Anwendung eines Wagegläschens. Die scharf geglühten Oxyde der seltenen Erdelemente haben durchaus nicht eine so stark ausgeprägte Hygroskopizität wie sie z. B. den wasserfreien Sulfaten eigen ist und was speziell die Wägegläschen betrifft, so sind wir der Ansicht, dafs man bei ibrer Verwendung sehr vorsichtig sein mufs; man mufs schon ein Opererateur wie z. B. Brauner sein, und mit den erforderlichen Hilfsmitteln ausgestattet sein, will man nicht statt der Verringerung der Fehler das umgekehrte erreichen.

Während des Erkaltens des Tiegels werden in einem vorher gewogenen, mit Porzellandeckel versehenen ErLenmeyerschen Kolben aus Jenaer Glas ca. $20 \mathrm{ccm} n / 2$ Schwefelsäure eingewogen. Der Inhalt des Platintiegels wird jetzt in den Kolben entleert und der leere Tiegel sofort zurückgewogen. Den Inhalt des Kolbens versetzt man mit etwa $20 \mathrm{ccm}$ reinen Wassers, von dessen absoluter Neutralität gegen Methylorange man sich vorher überzeugt hat. Jetzt wird das Oxyd, nötigenfalls unter Zufuhr von Wärme, in Lösung gebracht und nach dem Erkalten und Hinzufügen des Indikators die überschüssige Schwefelsäure mit $n / 10$ Natronlauge zurücktitriert. Als Vergleichsflüssigkeit dient reines Wasser, welches mit dem gleichen Quantum des Indikators versetzt wird. Bei rot gefärbten Lösungen, z. B. beim Neodym, dient als Vergleichsflüssigkeit eine mit Indikator versetzte Lösung von Neodymsulfat von annährend derselben Konzentration wie sie die zu titrierende Flüssigkeit besitzt. Wir nehmen mit Absicht nur einen geringen Überschuls an $n / 2$ Schwefelsäure, damit die Fehler, welche beim Zurücktitrieren durch Temperaturschwankungen entstehen können, auf ein Minimum reduziert werden.

Im folgenden geben wir die Resultate der auf diese Weise von uns vorgenommenen Atomgewichtsbestimmungen, soweit uns bis heute das zum Teil sehr kostbare Material dazu zur Verfügung stand.

In den Tabellen bedeutet

$a=$ Gewicht des Oxyds in Gramm,

$b=$ Zur Neutralisation erforderliche Schwefelsäure in Gramm, 
$c=$ Titer der Schwefelsäure, ausgedrückt in Gramm Sauerstoff pro Gramm Säure,

$d=$ gefundene Menge Sauerstoff im Oxyd,

$e=$ A tomgewicht.

\section{Lanthan.}

Das Lanthanmaterial wurde von uns dargestellt durch Umwandeln von schwach praseodymhaltigem Lanthanmagnesiumnitrat in das Ammoniumnitratdoppelsalz und Fraktionieren dieses Salzes, bis die gesättigte Lösung in $10 \mathrm{~cm}$ Dicke keine Spur eines Absorp. tionsspektrums mehr erkennen liefs. Das so angesammelte fast absolut reine Material wurde für sich noch einige Zeit derselben fraktionierten Kristallisation unterworfen und dann die am schwersten lösliche Fraktion durch zweimalige Fällung mit reiner Oxalsäure in das Oxalat verwandelt, welches nach dem Glühen im Porzellantiegel ein rein weifses Lanthanoxyd lieferte; dasselbe hat die für den vorliegenden Zweck besonders angenehme Eigenschaft, sich schon in der Kälte leicht in $n / 2$ Schwefelsäure aufzulösen.

\begin{tabular}{c|c|c|c|c|c}
\hline Nr. & $a$ & $b$ & $c$ & $d$ & $e$ \\
\hline 1 & 0.5125 & 18.954 & 0.0039801 & 0.07544 & 139.05 \\
2 & 0.5256 & 19.431 & 0.0039801 & 0.07731 & 139.11 \\
3 & 0.4835 & 17.878 & 0.0039801 & 0.07116 & 139.08 \\
4 & 0.5235 & 19.362 & 0.0039801 & 0.07706 & 139.04 \\
5 & 0.4815 & 17.810 & 0.0039801 & 0.07088 & 139.03 \\
6 & 0.5156 & 19.057 & 0.0039801 & 0.07585 & 139.15 \\
7 & 0.5348 & 19.766 & 0.0039801 & 0.07867 & 139.15 \\
& & & \multicolumn{3}{|c|}{ Atomgewicht im Mittel $=139.09$}
\end{tabular}

Der gefundene Wert von 139.09 ist genügend übereinstimmend mit dem Befunde von Brauner und PavLicek, nämlich 139.04.

\section{Praseodym.}

Die Darstellung des für unsere Atomgewichtsbestimmungen benutzten Praseodymsuperoxyds haben wir vor einiger Zeit ${ }^{1}$ genau beschrieben. Benutzt wurde die Demarcay-Drossbachsche Magnesiumnitratmethode bis zur völligen Entfernung des Neodyms, während die letzten Spuren von Lanthan durch Kristallisation

1 Z. anorg. Chem. 43, 205. 
der einfachen Nitrate eliminiert wurden. Es ist nun nicht leicht, ein von jeder Spur überschüssigen Sauerstoffs freies reines Praseodymoxyd zu erhalten, da die Darstellung des zur Reduktion des Superoxyds erforderlichen absolut reinen Wasserstoffs keine sehr leichte Sache ist. Wir hahen deshalb auf die Reduktion des Superoxyds verzichtet und dasselbe nach zweimaligem heftigen Glühen gewogen, in den ERLENMEYERschen Kolben mit der $n / 2$ Schwefelsäure zusammengebracht und den beim Erwärmen sich entwickelnden Sauerstoff, selbstverständlich unter Berücksichtigung aller hierzu erforderlichen Umstände, direkt mittels einer Hempeuschen Bürette gemessen. Das aus der Messung sich ergebende Gewicht an Sauerstoff wurde dann von der eingewogenen Menge Superoxyd in Abzug gebracht und ergab so die Menge des angewandten Oxyds.

\begin{tabular}{c|c|c|c|c|c|c|c}
\hline $\mathrm{Nr}$. & Superoxyd & Sauerstoff & $a$ & $b$ & $e$ & $d$ & $e$ \\
\hline 1 & 0.5528 & 0.01260 & 0.54010 & 19.797 & 0.0039801 & 0.07879 & 140.51 \\
2 & 0.5465 & 0.01230 & 0.53420 & $\mathbf{1 9 . 5 7 0}$ & 0.0039801 & 0.07789 & 140.60 \\
$\mathbf{3}$ & 0.5116 & 0.01106 & 0.50054 & 18.347 & 0.0039801 & 0.07302 & 140.51 \\
\hline
\end{tabular}

Atomgewicht im Mittel $=140.54$

Der von uns gefundene Wert für das Atomgewicht des Praseodyms ist somit fast identisch mit der von AuER v. WuLsbach gefundenen Zahl 140.57. V. Scheme findet 140.40, Jones gibt die Zahl 140.46 an, während Brauner den etwas höheren Wert 140.93 findet. Wir sind der Ausicht, dals der wahre Wert nicht weit von 140.5 abweicht.

\section{Neodym.}

Auch beim Neodym benutzten wir ein von uns durch Kristallisation der Magnesiumdoppelnitrate gewonnenes Material. Die Reinigung des Neodyms von einem Gehalte an Samarium ist nach dieser Methode relativ leicht zu bewerkstelligen, während die völlige Entfernung des Praseodyms sehr zeitraubend ist. Das von uns dargestellte Neodymoxyd war von rein himmelblauer Farbe. Nach unserer Ansicht ist diese Farbe das beste Kriterium für die Abwesenheit von Praseodym. Spuren des letzteren Elementes verursachen einen graublauen Ton, welcher bei etwas gröfserer Menge rötlich wird. Um beim Titrieren nicht durch die violette Farbe der Lösung getäuscht $z u$ werden, benutzten wir, wie schon erwähnt, als Vergleichs- 
lösung eine neutrale Neodymsulfatlösung von annähernd gleicher Konzentration.

\begin{tabular}{c|c|c|c|c|c}
\hline $\mathrm{Nr}$. & $a$ & $b$ & $e$ & $d$ & $e$ \\
\hline$=$ & 0.5380 & 19.249 & 0.0039801 & 0.07661 & 144.54 \\
1 & 0.5388 & 19.284 & 0.0039801 & 0.07675 & 144.48 \\
2 & 0.5358 & 19.176 & 0.0039801 & 0.07632 & 144.49 \\
3 & 0.5265 & 18.835 & 0.0039801 & 0.07497 & 144.56 \\
\hline
\end{tabular}

Atomgewicht im Mittel $=144.52$

Der gefundene Wert deckt sich fast genau mit dem von A tER angebenen, nämlich 144.54 .

\section{Samarium.}

Unser Samariumpräparat, welches ebenfalls durch Kristallisation der Magnesiumdoppelnitrate erhalten wurde, war zwar absolut frei von Neodym, enthielt aber, wie Herr Dr. EBERHARD uns mitzuteilen die Freundlichkeit hatte, eine geringe Menge Europium. Da jedoch Europium in den Monaziterden überhaupt nur in sehr geringer Menge vorkommt, wir aufserdem die am schwersten lösliche Doppelnitratfraktion benutzten, so kann der Gehalt an Europium $0.1 \%$ auf keinen Fall erreicht haben. Im übrigen erhöht ein Europiumgehalt von einem vollen Prozent das Atomgewicht des Samariums erst um 0.02 Einheiten.

\begin{tabular}{c|c|c|c|c|c}
\hline $\mathrm{N}$. & $a$ & $b$ & $e$ & $d$ & $e$ \\
\hline 1 & 0.5576 & 19.265 & 0.0039801 & 0.07668 & 150.53 \\
2 & 0.5576 & 19.272 & 0.0039801 & 0.07670 & 150.47 \\
3 & 0.5583 & 19.306 & 0.0039801 & 0.07684 & 150.38 \\
4 & 0.5633 & 19.464 & 0.0039801 & 0.07747 & 150.51 \\
\hline
\end{tabular}

Atomgewicht im Mittel $=150.47$

Der erbaltene Wert ist völlig identisch mit dem von URBaIN und LACOMBE am absolut reinem Material ermittelten Durchschnittswerte von 27 Bestimmungen.

\section{Europium.}

Durch die Liebenswürdigkeit des Herrn Dr. G. URBaIN, Paris, welcher uns eine Quantität reines, von EBERHARD ${ }^{1}$ als spektrosko-

1 2. anorg. Chem. 45, 374. 
pisch rein bezeichnetes Europiumoxyd übersandte, sind wir in die Lage versetzt worden, auch das Atomgewicht dieses Elementes zu bestimmen. Das Präparat ist von URBAIN durch Kristallisation der Magnesiumdoppelnitrate mit Hilfe des isomorphen Magnesiumwismuthnitrates aus den Mittelfraktionen zwischen Samarium und Gadolinium erhalten worden.

\begin{tabular}{c|c|c|c|c|c}
\hline Nr. & $a$ & $b$ & $e$ & $d$ & $e$ \\
\hdashline- & & & & & \\
\hline 1 & 0.3961 & 13.530 & 0.0039801 & 0.05385 & 152.54 \\
2 & 0.4096 & 13.984 & 0.0039801 & 0.05566 & 152.62 \\
3 & 0.4115 & 14.054 & 0.0039801 & 0.05594 & 152.56 \\
\hline
\end{tabular}

Atomgewicht im Mittel $=152.57$

Die Zahl 152.57 weicht von dem Durchschnitte der UndaINschen Bestimmungen 151.94 um 0.63 Einheiten ab. Die Differenz ist also recht exheblich; den Grund dafür vermögen wir nicht anzugeben.

\section{Gadolinium.}

Auch ein spektroskopisch reines Gadoliniumoxyd stelite uns Herr Dr. URbain zur Verfügung; wir wollen nicht verfehlen, dem französischen Gelehrten, welcher sich um die Erforschung der seltenen Erdén so aufserordentlich grolse Verdienste erworben hat, unseren verbindlichsten Dank auszudrücken. Wir nehmen an, dals das Präparat durch Kristallisation der Nickeldoppelnitrate gewonnen wurde. Es ist bemerkenswert, dafs die Lösung des Gadoliniumoxyds in der $n / 2$ Schwefelsäure schon etwas schwieriger vor sich geht; in dieser Beziehung ist ein ganz allmählicher Übergang von der stärksten zur schwächsten Base zu bemerken.

\begin{tabular}{|c|c|c|c|c|c|}
\hline Nr. & $a$ & $b$ & $c$ & $d$ & $e$ \\
\hline 1 & 0.3852 & 12.865 & 0.0039623 & 0.05097 & 157.37 \\
\hline 2 & 0.3956 & 13.210 & 0.0039623 & 0.05234 & 157.40 \\
\hline
\end{tabular}

Atomgewieht im Mittel $=157.38$

Die Übereinstimmung mit der UnbaINschen Zahl 157.24 ist also eine genügende.

In der Reihe der Elemente der seltenen Erden würden nun noch in Betracht kommen das Terbium, Dysprosium, Holmium, Erbium, Thulium, Ytterbium und Yttrium. Von diesen sieben Ele- 
menten sind die beiden ersten, Terbium und Dysprosium, in reinem Zustande, und zwar ebenfalls von URBaIN, dargestellt worden; Holmium, Erbium und 'Thuliumpräparate existieren noch nicht in völliger Reinheit, dagegen sind die beiden letzten, Ytterbium und Yttrium, leicht zugänglich.

\section{Ytterbium.}

Über die Darstellung des Ytterbiummateriales werden wir in einer späteren Abhandlung berichten. Das von uns zur Bestimmung des Atomgewichtes benutzte Oxyd war rein weils; die Lösung liefs keinerlei Absorptionsstreifen erkennen; es konnte demnach wesentliche Mengen von Thulium nicht enthalten. Wir können die Bemerkung von Astrid Cueve ${ }^{1}$ bestätigen, dafs Spuren von Thulium das Ytterbiumoxyd gelblich färben. Scandium konnte ebenfalls nicht vorhanden sein, weil durch eine weitere Zerlegung des Materiales keine schwächere Base mit geringerem Atomgewichte sich abscheiden liefs. Wir halten daher unser Material, vielleicht abgesehen von einer minimalen Spur Thulium, für rein; da ein volles Prozent Thulium das Atomgewicht nur um 0.02 Einheiten erniedrigt, so ist das Material für den vorliegenden $Z$ weck als genügend rein zu betrachten.

Das Ytterbiumoxyd löst sich nur langsam in verdünnter Schwefelsäure auf. Wir haben deshalb in diesem Falle die Säure nicht verdünnt, sondern das $0 x y d$ mit der $1 / 2$ normalen Säure einige Stunden bei etwa $90^{\circ}$ unter öfterem Umschütteln stehen gelassen, bis völlige Lösung stattgefunden. Zuweilen findet sich noch nach mehrstündigem Digerieren ein Quantum von einigen Zehntel Milligrammen bis zu einem Milligramm ungelöst; diese Bestimmungen wurden verworfen.

\begin{tabular}{c|c|c|c|c|c}
\hline $\mathrm{Nr}$. & $a$ & $b$ & $o$ & $a$ & $e$ \\
\hline 1 & 0.6424 & 19.617 & 0.0039802 & 0.07808 & 173.46 \\
2 & 0.6408 & 19.554 & 0.0039802 & 0.07783 & 173.60 \\
3 & 0.6403 & 19.544 & 0.0039802 & 0.07779 & 173.55 \\
4 & 0.6466 & 19.743 & 0.0039802 & 0.07858 & 173.48 \\
\hline
\end{tabular}

Atomgewicht im Mittel $=173.52$

Auch diese Zahl weicht nicht wesentlich von den bisherigen Bestimmungen von Nilson, A. Clieve und Bradner, deren Mittel 173.1 ist, ab.

\footnotetext{
1Z. anorg. Chem. 32, 134.
} 


\section{Yttrium.}

Für die Bestimmung des Atomgewichtes des Yttriums stand uns ein Präparat von Posrruus zur Verfügung, welches wir dem liebenswürdigen Entgegenkommen des Herrn Prof. Dr. Muthmann, München, verdanken. Nach den Angaben von EBERHARD ist dieses Präparat spektroskopisch rein. Aufserdem haben wir ein Präparat eigener Darstellung benutzt. Als Ausgangsprodukt verwandten wir ein durch Kombination mehrerer bekannter Methoden dargestelltes Präparat mit dem Atomgewicht 89.6. Herr Dr. EBERHARD in Potsdam hatte die grolse Liebenswürdigkeit, dieses Yttriumoxyd spektroskopisch zu untersuchen. Es stellte sich bei der Untersuchung heraus, dafs das Präparat von allen benachbarten Erden absolut frei war, jedoch nicht unbeträchtliche Mengen von Lanthan enthielt. Mehrere Versuche, das Atomgewicht herunterzudrücken, mif́slangen. Wir entschlossen uns daher, die Chromatmethode von Mutemann und BöнM anzuwenden, da bei dieser das Lanthan sich von allen Erden zuerst abscheidet, während das Yttrium eines der leichtest löslichen Chromate bildet. Die erste Fraktion besals ein Atomgewicht von 89.82, die zweite ergab die Zahl 89.51, die dritte 89.32, eine Zahl, welche auch nach zwei weiteren Fraktionen nicht erniedrigt werden konnte. Wir waren daher zu der Annahme berechtigt, dafs das Präparat rein sei; die Farbe des Oxyds war rein weifs.

Die Bestimmungen ergaben folgende Zahlen:

Yttriumoxyd ron Postius:

\begin{tabular}{|c|c|c|c|c|c|}
\hline $\mathrm{Nr}$. & $a$ & $b$ & $c$ & $d$ & $e$ \\
\hline 1 & 0.3677 & 19.625 & 0.0039647 & 0.07781 & 89.42 \\
\hline 2 & 0.4928 & 26.327 & 0.0039647 & 0.10438 & 89.31 \\
\hline 3 & 0.3660 & 19.470 & 0.0039801 & 0.07749 & 89.36 \\
\hline
\end{tabular}

Atomgewicht im Mittel $=89.36$

Yttriumoxyd eigener Darstellung:

\begin{tabular}{c|c|c|c|c|c}
\hline $\mathrm{Nr}$. & $a$ & $b$ & $c$ & $d$ & $e$ \\
\hline \hline 4 & 0.3660 & 19.474 & 0.0039801 & 0.07751 & 89.33 \\
5 & 0.3704 & 19.698 & 0.0039801 & 0.07840 & 89.39 \\
6 & 0.3635 & 19.348 & 0.0039801 & 0.07701 & 89.29 \\
\hline
\end{tabular}

Atomgewicht im Mittel $=89.34$ 
Die neueren Bestimmungen des Atomgewichtes des Yttriums schwanken zwischen 88.45 und 89.11 und zwar sind gerade die letzten Bestimmungen die niedrigsten. Es könnte nun den Anschein haben, als ob der Grund allein darin läge, dafs die späteren Autoren ein immer reineres Präparat in Händen gehabt hätten. Wir halten es jedoch für ausgeschlossen, dals Forscher wie Cleve, Marignac und Jones ein Yttriumoxyd mit so erheblichen Verunreinigungen benutzt haben, wie sie hier in Frage kommen würden. Nehmen wir als verunreinigenden Bestandteil das Lanthan, also das Element mit nächst höherem Atomgewichte an, so müfste, da das Präparat ron Postros [Atomgewicht $=88.45$ ] bestimmt rein gewesen ist, z. B. das Yttriumoxyd von Cleve [Atomgewicht $=89.11$ ] mindestens $1.3 \%$ Verunreinigungen enthalten haben; das Marignacsche Yttriumoxyd [Atomgewicht $=88.88$ ] mülste über $0.8 \%$ andere Oxyde enthalten. Die Annahme ist also wohl gerechfertigt, dafs die Differenzen im wesentlichen in der Bestimmung des Atomgewichtes zu suchen sind; eine Annahme, deren Richtigkeit dadurch gestärkt wird, dafs wir mit dem Yttriumoxyde von Postids eine wesentlich höhere, mit dem unsrigen Präparate übereinstimmende Zahl erhielten. Vielleicht sind gerade bei der Atomgewichtsbestimmung des Yttriums nach der Sulfatmethode die oben erwähnten Fehler am schwersten zu vermeiden.

Die von uns erhaltenen Zahlen für die Atomgewichte einer Anzahl von Elementen der seltenen Erden sind für

\begin{tabular}{|c|c|c|c|c|c|}
\hline Lanthan & - . & - & & 139.09 & 139.17 \\
\hline Praseodym & . . & . & - & 140.54 & 140.62 \\
\hline Neodym & . . & 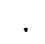 & . & 144.52 & 144.60 \\
\hline Samarium & . . & . & . & 150.47 & 150.56 \\
\hline Europium & . & . & . & 152.57 & 152.66 \\
\hline Gadolinium & . & . & . & 157.38 & 157.47 \\
\hline Ytterbium . & . . & . & - & 173.52 & 173.52 \\
\hline Yttrium & . . & . & • & 89.35 & 89.40 \\
\hline
\end{tabular}

Die Zahlen der ersten Reihe sind die direkt gefundenen; die zweite Reihe gibt die Zahlen reduziert auf den luftleeren Raum. Diese Reduktion ist für die Einstellung der Säure (Wägung von Natriumkarbonat mit Aluminiumgewichten) fast absolut belanglos; für die eigentlichen Bestimmungen (Wägung der Oxyde mit Aluminiumgewichten) beträgt die Korrektur beim Yttrium +0.05 , beim Lanthan, Praseodym und Neodym +0.08 und beim Samarium, Europium und Gadolinium + 0.09. Für das Ytterbium fällt die Kor- 
rektur fort, da wir das Ytterbiumoxyd mit Messinggewichten gewogen haben.

Was nun die Genauigkeit einer einzelnen Bestimmung des Atomgewichtes in der beschriebenen Weise betrifft, so würden, unter der Voraussetzung, dals der Titer der Säure durch eine Reihe von Bestimmungen genau festgelegt worden ist, die zwei für die Ermittelung der Menge des angewandten Oxyds erforderlichen Wägungen bei $0.1 \mathrm{mg}$ Empfindlichkeit der Wage im ungünstigsten Falle um $0.2 \mathrm{mg}$ von der Wirklichkeit abweichen. Dieser Fehler entspricht in unserem Falle \pm 0.05 Einheiten. Beim Zurücktitrieren mit Natronlauge schätzen wir die Unsicherheit auf 2 Tropfen unserer Bürette, d. h. auf einen Tropfen zu viel oder einen Tropfen zu wenig. Die Tropfen der angewandten Bürette hatten ein Volumen von $1 / 30 \mathrm{ccm}$. Der Fehler erreicht darnach rechnungsmälsig beim Yttrium \pm 0.07 , beim Ytterbium \pm 0.13 Einheiten. Wenn sich nun die beiden genannten Fehler addieren, so kann die Ungenauigkeit beim Yttrium \pm 0.12 und beim Ytterbium \pm 0.18 Einheiten betragen. Es würde dies der extremste Fall bei einer Einzelbestimmung sein. Es kann darnach wohl behauptet werden, dafs die oben von uns gefundenen Zahlen ganz erheblich genauer sind.

Durch die von uns auf volumetrischem Wege ausgeführten Atomgewichtsbestimmungen, welche in Anbetracht der uns zur Verfügung stehenden Hilfsmittel sich naturgemäls nicht vergleichen lassen mit solchen Bestimmungen, welche von berufeneren Seiten mit allem erforderlichen Raffinement bewerkstelligt werden, glauben wir gezeigt zu haben, dals diese einfache Methode in beachtenswerter Weise die Schwierigkeiten, welche sich der Atomgewichtsbestimmung der Elemente der seltenen Erden bisher entgegenstellten, vermeidet; wir sind deshalb der Ansicht, dals die Methode bei ihrer Anwendung für die exakte Bestimmung der Atomgewichte mit relativ geringer Mühe zu äufserst genauen Resultaten führen wird.

Vienenburg a/Harn, Laboratorium der Königl. Berginspeltion, Juli 1906.

Bei der Redaktion eingegangen am 30. Juli 1906. 